Wavelengths of the 3p-3d Transitions if the Co- and

Fe-Like Ions: The Effects of Electron Correlation

\author{
Mau Hsiung Chen \\ Lawrence Livermore National Lahoratory \\ University of California \\ Livermore, CA 94550 \\ U.S.A.
}

UCRL--97325

DEB8 000219

\title{
DISCLAIMER
}

This report was prepared as an account of work sponsored by an agency of the United States Government. Neither the United States Government nor any agency thereof, nor any of their employees, makes any warranty, express or implied, or assumes any legal liability or responsibility for the accuracy, completeness, or usefulness of any information, apparatus, product, or process disclosed, of represents that its use would not infringe privately owned rights. Reference herein to any specific commercial product, process, or service by trade name, trademark, manufacturer, or otherwise does not necessarily constitute or imply its endorsement, recommendation, of favoring by the United States Government or any agency thereof. The views and opinions of authors expressed herein do not necessarily state or reflect those of the United States Government or any agency thereof. 


\begin{abstract}
The experimental observations of the $3 p^{6} 3 d^{9} 2_{D}-3 p^{5} 3 d^{10}{ }^{2} P$ transitions of the Co-like ions and $3 p^{6} 3 d^{8}{ }^{3} F_{4}-3 p^{5} 3 d^{9} 3 F_{3}$ of the $F e-l i k e$ ions have recently been extended to highly charged ions of heavy elements up to uranium $(Z=92)$. A comparison between the observed energies and calculated values from the Dirac-Fock model indicated persistent discrepancies of 3-4 eV for all ions. Systematic multiconfiguration Dirac-Fock calculations for these transitions have been carried out with emphasis on the effects of electron correlation. The previously found discrepancies between theory and experiment have mostly been removed after the inclusion of the electron-electron correlation effects in the theoretical calculations.
\end{abstract}

\title{
1. INTRODUCTION
}

The spectra of multiple-charged ions are of great interest in connection with astrophysics. collision physics and laser produced plasmas. They also provide important information in testing atomic structure theory. The experimental observations of the $3 p^{6} 3 d^{9}$ ${ }_{2} D-3 p^{5} 3 d^{10} 2 p$ transitions of the Co-like ions and the $3 p^{6} 3 d^{8} 3 F_{4}-3 p^{5} 3 d^{9}{ }_{3} F_{3}$ of the Fe-like ions have recently been extended to highly charged ions of heavy elements up to uraniurn $(Z=92)$. A comparison between the observed energies and calculated values using single configuration Dirac-Fock (DF) wavefunctions revealed persistent discrepancies of 3-4 $\mathrm{eV}$ for all ions [1]. To remedy this situation, we have performed systematic multiconfiguration Dirac-Fock (MCDF) [2,3] calculations for the $3 p^{6} 3 d^{9}-3 p^{5} 3 d^{10}$ transition of the Co-like ions and the $3 p^{6} 3 d^{8} 3 F_{4}-3 p^{5} 3 d^{9} 3 F_{3}$ of the $\mathrm{Fe}$ isoelectronic sequences. We pay special attention to the effects of electron-electron correlation on the transition energies. The initial account for the Co-like ions has been published recently [4].

\section{THEORETICAL METHOD}

Multiconfiguration Dirac-Fock model is one of the most efficient and accurate method in calculations of atomic structure for many electron mediurn-heavy and heavy atoms. In the 
present work, we use the version of the MCDF model due to Grant et al. [2]. In the MCDF model [2], an atomic state function for a state $i$ with total angular momentum JM is expanded in terms of n configuration state functions (CSF):

$$
\Psi_{i}(J M)=\sum_{\lambda=1}^{n} C_{i \lambda} \phi\left(\Gamma_{\lambda} j M\right) \quad,
$$

where $C_{i \lambda}$ are the mixing coefficients for state $i$ and the CSF functions, $\phi\left(\Gamma_{\lambda} \mathrm{JM}\right)$, are formed by taking linear combinations of Slater determinants of the Dirac orbitals. The energy of an atomic state is given by the expectation value of the Hamiltonian. The Dirac-Fock equations are obtained by applying the variation principle to the energy functional and a self-consistent field (SCF) method is then used to solve the MCDF equations.

To determine accurately the transition energies, one needs to include the relaxation effect, finite-nuclear-size effect, Breit interaction, and quantum-electrodynamic correlations (QED) and electron correlation effect [5]. In the present work, the Breit energies were included through the use of the transverse Breit operator [3.6] in first order perturbation theory. The vacuum-polarization corrections were determined by evaluating the Uehling potential [3] and the self-energy corsections were calculated from point-Coulomb values [7] with an effective-charge screening procedure [3]. The finite nuclear size effect was taken into account by using uniform charge distribution. The relaxation effects were included by performing separate SCF calculations for the initial and final states and taking the energy difference to determine the transition energies.

To estimate the correlation corrections, we assume the correlation energies of the passive electrons from the initial and final states to cancel each other completely. We only need to deal with the residual correlation corrections which pertain to the characteristic of the initial and final states. One of the important correlation corrections is the dynamic relaxation processes due to the [3p] $\leftrightarrow\left[3 \mathrm{~d}^{2}\right]$ nf super-Coster-Kronig fluctuation $[5,8]$. Here [3l] denotes a hole in the $3 l$ subshell. In the present work, the $[3 p] \leftrightarrow\left[3 d^{2}\right]$ nf interaction was taken into account by using the MCDF model with the optimal level (OL) procedure [2]. The CSF functions included for the initial state of the Co-like ions are $3 p^{5} 3 d^{10}, 3 p^{6} 3 d^{8} 4 f$ and $3 p^{6} 3 d^{8} 4 p$ and those for the initial state calculations of the Fe-like ions are the states from the $3 p^{5} 3 d^{9}$ and $3 p^{6} 3 d^{7} 4 f$ configurations. For the $3 p^{6} 3 d^{9}$ and $3 p^{6} 3 d^{8} 3 F_{4}$ final states, single configuration Dirac-Fock calculations were performed. For comparison we also carried out single configuration DF calculations for the initial states.

The other important correlation correction is the residual all-external pair correlation correction $[5,8,9]$. This correlation correction arises from the difference in the all-external pair energies between the initial and final states. For the $3 p^{6} 3 d^{9}-3 p^{5} 3 d^{10}$ and the $3 p^{6} 3 d^{8}$ ${ }^{3} F_{4}-3 p^{5} 3 d^{9} 3 F_{3}$ transitions, the residual all-external pair correlation corrections have been estimated to be -1.41 and $-1.16 \mathrm{eV}$, respectively for all ions by using the pair energies calculated for the $\mathrm{Zn}^{2+}$ ion. [10]. For details, the reader is referred to Ref. 4.

\section{RESULTS AND DISCUSSION}

The wavelengths for the $3 p^{6} 3 d^{9} 2 D_{J}-3 p^{5} 3 d^{10} 2 p_{J}$ transitions of the Co-like ions and the $3 p^{6} 3 d^{8} 3_{F_{4}}-3 p^{5} 3 d^{9} 3_{F_{3}}$ transitions of the Fe-like ions were evaluated by using MCDF-OL method [2] for ions with atomic number $38 \leq Z \leq 92$. The results for the Fe isoelectronic sequence are listed in Table I.

For the 3p-3d transitions, the QED corrections and the finite-nuclear size effects are quite small. The Breit interaction reduces the transition energies by 3-4 eV for heavy ions. The correlation corrections remain fairly constant $(\sim-3 \mathrm{eV})$ for all ions.

The calculated transition energies for the $3 p^{6} 3 d^{9}{ }^{2} D_{5 / 2}-3 p^{5} 3 d^{10} 2 p_{3 / 2}$ transitions of the Co-like ions and $3 p^{6} 3 d^{8} \quad 3_{F_{4}}-3 p^{5} 3 d^{9} 3_{F_{3}}$ transitions of the $F e-l i k e$ ions are compared with experiments [1,11-13] in Figs. 1-2. The single configuration DF calculations overestimate the transition energies by $-3-4 \mathrm{eV}$. The previously found discrepancies between theory and experiment have mostly been removed due to the inclusion of the electron 
correlation effects in the theoretical calculations. The remaining discrepancies are probably due to the inadequacy in the treatment of the electron correlation especially for the residual all-external pair correlation correction.

Table I. Calculated wavelengths in angstroms for the transition $3 p^{6} 3 d^{8}{ }^{3} F_{4}-3 p^{5} 3 d^{9}{ }^{3} F_{3}$ of the Fe-like ions.

A.tomic No.

Wavelength

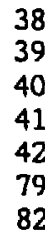

39

40

41

42

79

82

83
92.869

88.155

83.865

79.944

76.345

24.147

22.382

21.825
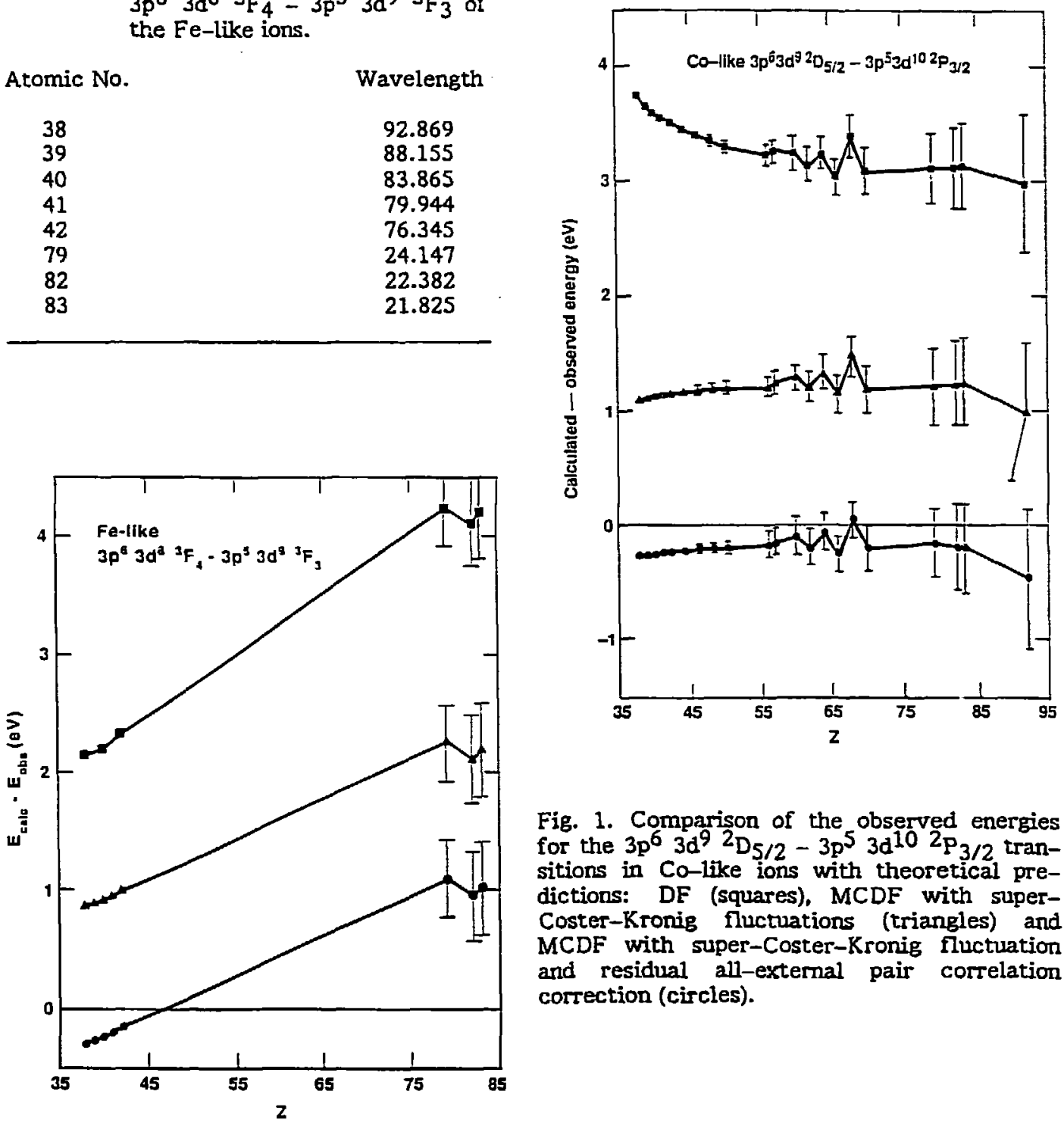

Fig. 1. Comparison of the observed energies for the $3 p^{6} 3 d^{9}{ }^{2} D_{5 / 2}-3 p^{5} 3 d^{10} 2_{3 / 2}$ transitions in Co-like ions with theoretical predictions: DF (squares), MCDF with superCoster-Kronig fluctuations (triangles) and MCDF with super-Coster-Kronig Iuctuation and residual all-external pair correlation correction (circles).

Fig. 2. The same as Fig. 1 except for the $3 p^{6}$ $3 d^{8}{ }^{3} F_{4}-3 p^{5} 3 d^{9} 3 F_{3}$ transitions of the $F e-$ like ions. 
Work performed under the auspices of the U.S. Department of Energy by the Lawrence Livermore National Laboratory under contract number W-7405-ENG-48.

\section{REFERENCES}

[1] Seely, J. F., Ekberg, J. O., Brown, C. M., Feldman, U., Behring, W. E., Reader, J. and Richardson, M. C., Phys. Rev. Lett. 57 (1986) 2924.

[2] Grant, I. P., McKenzie, B. J., Norrington, P. H., Mayers, D. F. and Pyper, N. C., Comput. Phys. Commun. 21 (1980) 207.

[3] McKenzie, B. J., Grant, I. P. and Norrington, P. H., Comput. Phys. Commun. 21 (1980) 233.

[4] Chen, M. H., Phys. Rev. A 36 (1987) 665.

[5] Chen, M. H., Crasemam, B., Martensson, N. and Johansson, B., Phys. Rev. A 31 (1985) 556.

[6] Mann, J. B. and Johnson, W. R., Phys. Rev. A. 4 (1971) 41.

[7] Mohr, P. J.. Ann. Phys. (N.Y.) 88 (1974) 52; Phys. Rev. Lett. 34 (1975) 1050; Phys. Rev. A 26 (1982) 2338.

[8] Ohno, M. and Wendin G., J. Phys: B 11 (1979) 1557.

[9] öksiiz, I. and Sinanoglu, O., Phys. Rev. 181 (1969) 42; 181 (1969) 54.

[10] Jankowski, K., Malinowski, P. and Polasik. M., J. Chem. Phys. 76 (1982) 448.

[11] Reader, J. and Ryabtsev, A., J. Opt. Soc. Am. 71 (1981) 231.

[12] Ryabtsev, A. N. and Reader, J., J. Opt. Soc. Am. 72 (1982) 710.

[13] Reader, J., J. Opt. Soc. Am. 73 (1983) 63. 